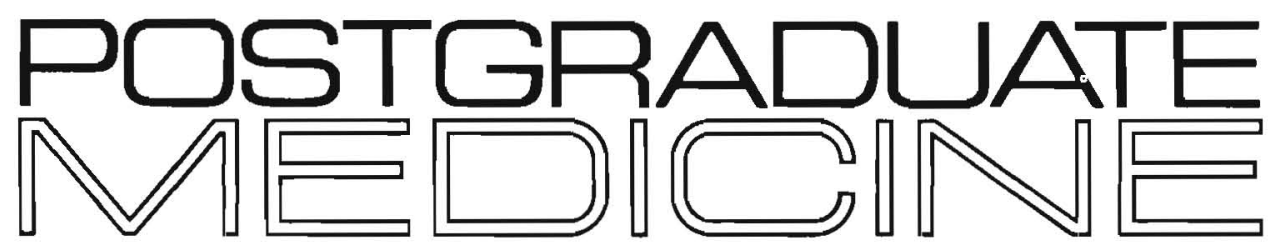


CARL G. GROTH, M.D.

THOMAS E. STARZL, M.D., Ph.D.

University of Colorado School of Medicine Denver $\uparrow$ By May 1972, 60 patients had undergone orthotopic or auxiliary hepatic transplantation at the University of Colorado Medical Center and the Veterans Administration Hospital, Denver. A number of these patients benefited from the operation

\section{Liver Transplantation}

Sixty patients with severe liver disease have undergone hepatic transplantation in Denver. Deterrents to success have been mechanical vascular and biliary complications, graft rejections, severe infections, and recurrence of hepatic malignancy. Nonetheless, $I I, 4$ and 2 of the patients have survived beyond one, two and three years, respectively. Avenues for further improvement are discussed. through, at least, temporary return to a normal life. This report presents the indications, the problems of management, and the results.

\section{The Patients}

In all instances the indication for the transplantation was life-threatening liver disease for which no other form of treatment was available. Initially, nonresectable malignant liver tumors were believed to be an important indication for total liver replacement. However, the high incidence of tumor recurrence has prompted a moratorium in transplantation of the liver in these cases.

Among the nonneoplastic diseases, biliary atresia has been the most common single indication for liver transplantation. More recently the number of patients with cirrhosis and chronic aggressive hepatitis undergoing the operation has increased. When the first attempts were made to replace the liver in patients with benign liver disease, the recipients were in end-stage hepatic failure and gastrointestinal bleeding, hepatic coma, or renal insufficiency usually was present. Obviously their poor condition reduced the chances of success. 
Recently an attempt has been made to operate before the advent of this procession of terminal complications.

Because of the high proportion of patients with biliary atresia, about 45 percent of the recipients have been younger than

\section{in Man}

five years of age. No other organ transplantation has been carried out in such a high proportion of small children.

All the hepatic homografts have been obtained from cadaveric donors. In more than a dozen of the first cases, in situ cooling and removal of the liver were begun after cardiac arrest had occurred. Since the acceptance of the brain death criteria in 1968, the grafts have been obtained without such an ischemic insult. At the same time it has become possible to delay the removal of the graft until completion of the recipient hepatectomy, a condition that obviates the need for ex vivo graft preservation measures.

Until 1969 an attempt often was made to select donor-recipient combinations on the basis of HL-A histocompatibility. Since then the results of such matching have been found not to correlate with clinical results in recipients of nonrelated renal and other kinds of homografts ${ }^{1,2}$ and this kind of effort has been deemphasized. Present requirements for a donor include only a compatible $A B O$ blood group and absence of malignancy or generalized sepsis. Reasonable similarity in size of donor and recipient is desirable.

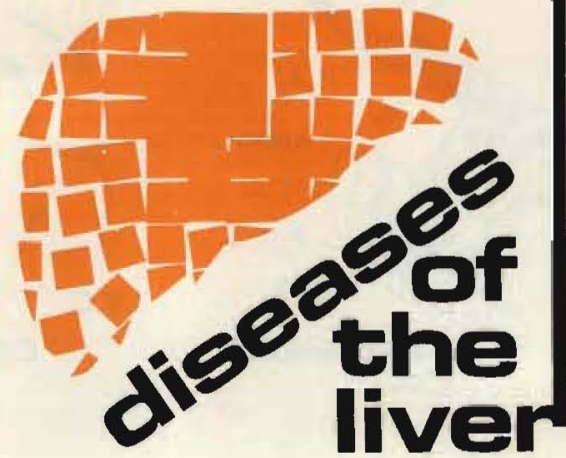

\section{The Transplantation}

Orthotopic transplantation (total replacement of the liver with anatomic reconstruction of the vasculature) was carried out in 56 of the 60 patients (figure 1). The necessary cross-clamping of vena cava and portal vein is tolerated for at least 90 minutes. In the remaining four patients (all of whom had nonneoplastic liver disease), the patient's own liver was left in place and the graft was inserted in a heterotopic position (figure 2). However, the possible advantages of retaining the residual function of the diseased liver are offset by several factors. Portal hypertension remains unrelieved unless a shunt procedure is performed. The diseased liver may continue to be a threat to life as the site of hepatoma; this was observed as an incidental finding in specimens from two patients in our series. The abdominal overcrowding has led to a very high incidence of pulmonary complications. Finally, residual function in a host liver may enable it, by a process loosely termed interliver competition, to lessen the chances of good function and survival of the transplant. ${ }^{3}$

While portal or caval vascular complications have not been common, in five patients fatal hepatic arterial thrombosis de- 


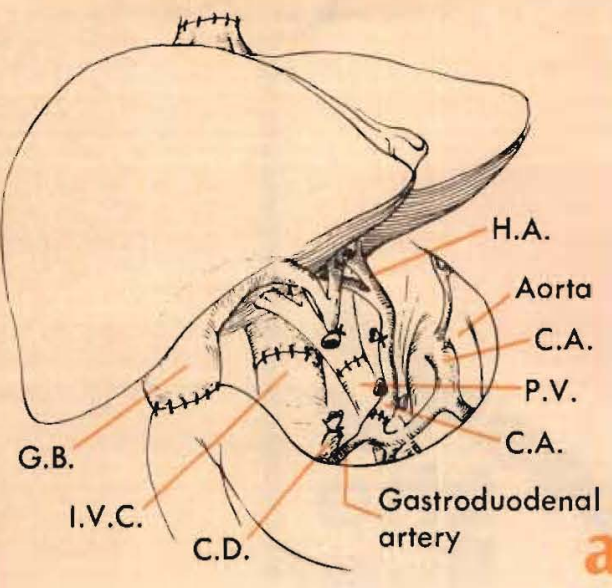

Figure 1. Orthotopic hepatic transplantation.

a. Anatomic reconstruction of inferior vena cava above and below the liver and of the portal vein and the hepatic artery. Biliary drainage is with cholecystoduodenostomy after ligation of the graft as well as the patient's common duct.
Anomalous Hepatic artery to artery to

G.B. right lobe

left lobe
I.V.C.

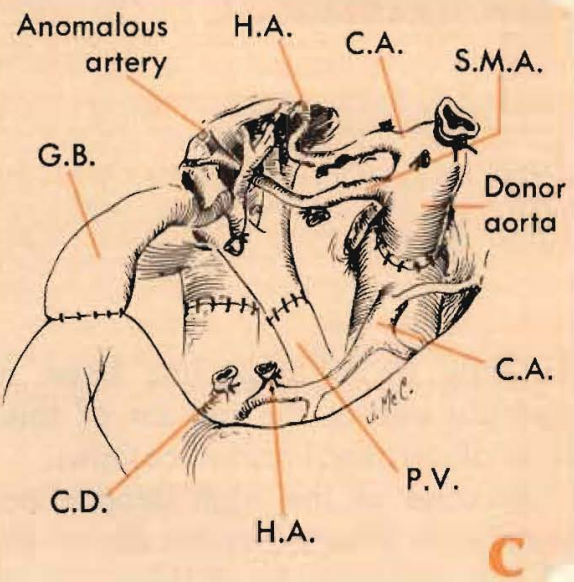

C.A.

b, c. Arterial reconstructions used in two patients when homograft had a double arterial blood supply: two anastomoses (b) and anastomosis of donor aorta to recipient aorta (c). Several other variations have been necessary in other patients.

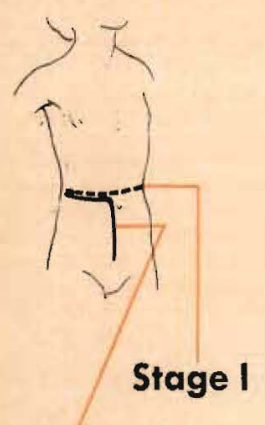

Stage II

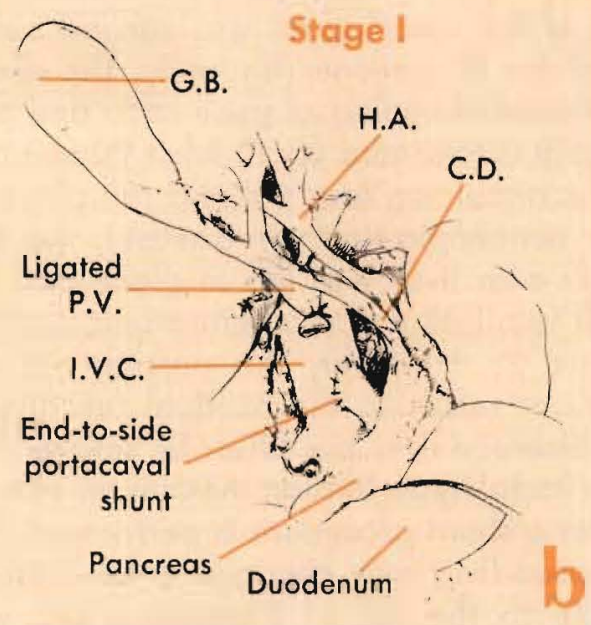

Figure 2. Heterotopic hepatic transplantation.

a. The incisions.

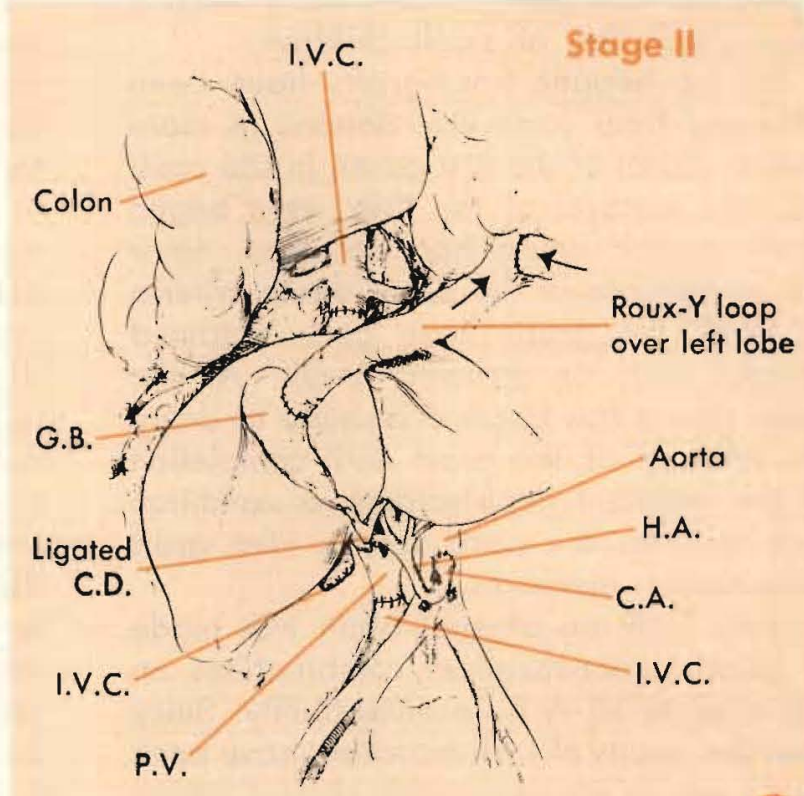

b. Portacaval shunt performed before transplantation.

c. The hepatic graft revascularized in the lower abdomen. Portal inflow is from the patient's inferior vena cava $;$ the inferior end of the graft vena cava has been closed. Biliary drainage is with cholecystoenterostomy to a Roux-Y loop.

In other patients, the portal vein has been anastomosed to the splenic or mesenteric vein to provide inflow of splanchnic blood into the graft.

Reproduced from Halgrimson CG, et al: Arch Surg 93:107, 1966. Copyright 1966, American Medical Association. 
veloped immediately after operation or within a few weeks. This complication was seemingly related to the small size of the vessels, especially in children, and to arterial anomalies with hepatic branches emerging from the superior mesenteric or other anomalous sources. The multiple effects of the liver on blood coagulation might have been a contributing factor.

In the majority of the patients the reconstruction of the biliary tract has been with anastomosis of the graft gallbladder to the recipient duodenum (figure 1). Although obstruction caused by technical or infectious complications involving the cystic duct has occurred in six of these patients, ${ }^{3,4}$ this method of reconstruction remains the preferred procedure, as it does not unnecessarily sacrifice potentially useful tissue. The method carries a very low incidence of bile leakage, and in the event of malfunction, a secondary procedure can be performed either by implanting the common bile duct in the duodenum or by end-to-end anastomosis of the graft and host common ducts.

\section{Early Postoperative Management}

The implantation of a liver damaged by ischemia is followed by hypoglycemia, severe clotting abnormalities, and development of a third fluid space with severe electrolyte and acid-base disturbances. ${ }^{3}$ However, the early course after implantation of a minimally injured liver usually is not much different from what is seen following other kinds of major abdominal surgery. Careful monitoring of arterial blood gases and of serum potassium and glucose, with swift and appropriate adjustments in therapy, is important. Most patients in our experience have benefited from intravenous administration of albumin and diuretics over the first several postoperative days. Vitamin $K$ is given daily to support the synthesis of clotting factors. A triple combination of antibiotics effective against a broad spectrum of Gram-negative and Gram-positive bacteria is given during the first postoperative weeks.

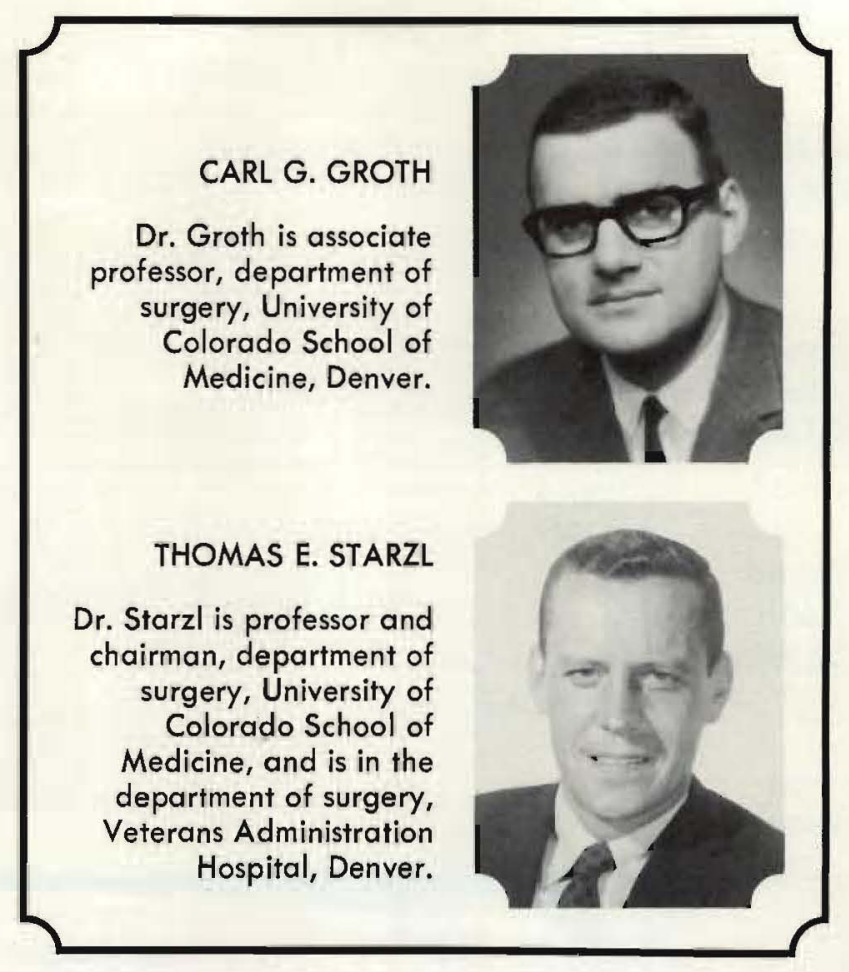

Graft Rejection and Immunosuppression

Some animals, particularly the pig and the baboon, receiving hepatic homografts have survived for long periods without immunosuppression, and it has been concluded that the liver enjoys a privileged immunologic status in these species. Such a situation also may exist in man, but if so, any advantage is minor. Occurrence of rejection episodes in human recipients of hepatic homografts is the rule rather than the exception:

So far, no absolutely specific tests for diagnosis of hepatic homograft rejection have become available. The most characteristic changes in the standard liver function tests include an increase in serum bilirubin and in alkaline phosphatase paralleling concomitant intrahepatic cholestasis. If rejection progresses, rises in serum transaminases, prolongation of prothrombin time, and other indications of cellular injury eventuate (figure 3 ).

The biochemical tests poorly differentiate graft rejection from other pathologic 


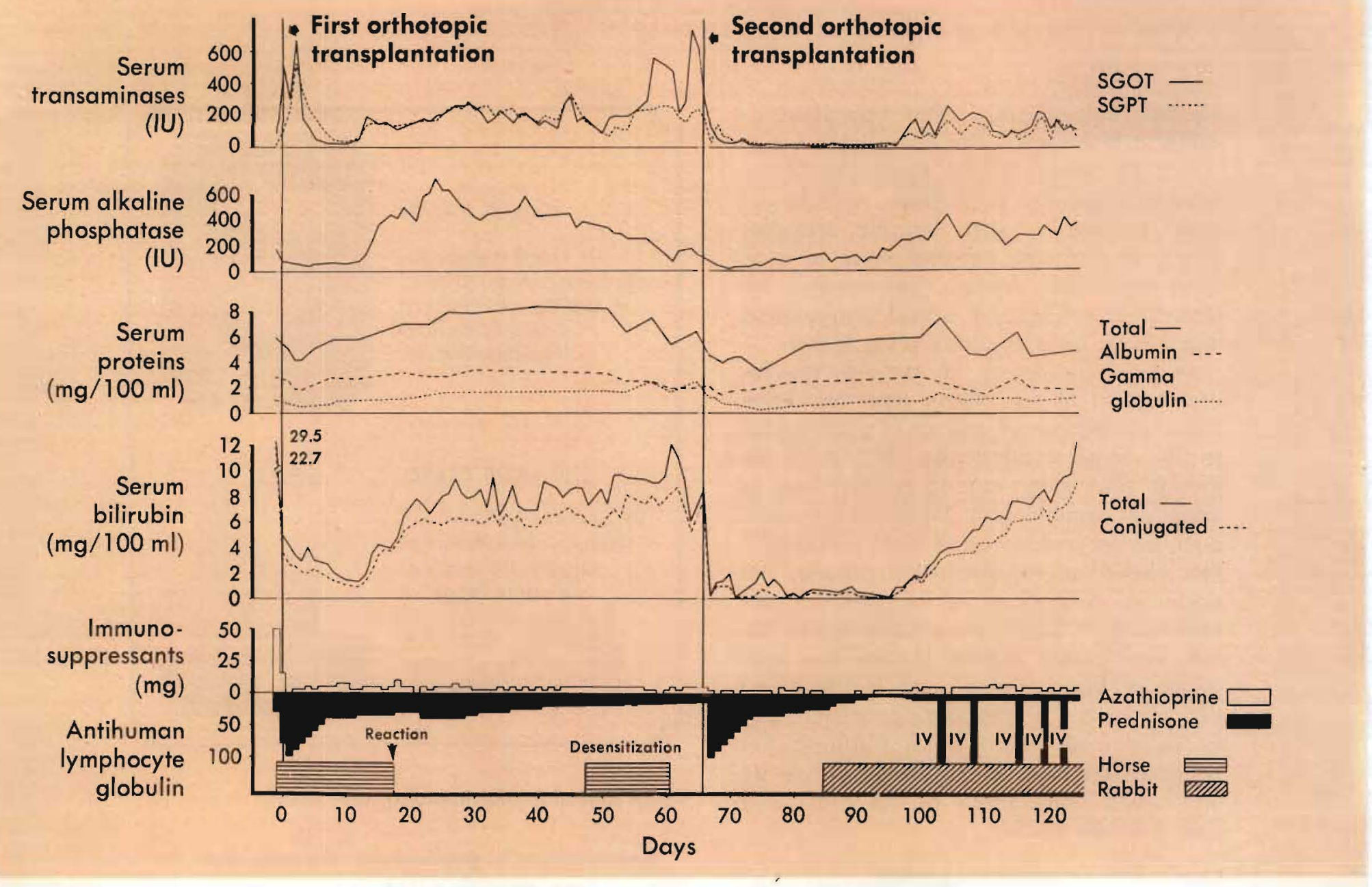

Figure 3. Course of a two year old child with biliary atresia. The first hepatic graft was rejected in two months. Note the hyperbilirubinemia and the marked elevation of alkaline phosphatase accompanying a moderate increase in serum transaminases.

After the second hepatic transplantation, graft function was excellent at first. Later there was evidence of acute rejection, but this time the process could be reversed. The patient lived another 12 months. Immunosuppression was with azathioprine, prednisone, and horse and rabbit antihuman lymphocyte globulin.

processes such as biliary obstruction, infectious hepatitis, and hepatic injury caused by drugs. Intravenous cholangiograms, technetium and rose bengal radioisotope scans, needle biopsy, and serum testing for Australia antigen all can aid in the differential diagnosis.

The extensive experience with renal transplantation provides the foundation for the immunosuppressive regimens used in human hepatic transplantation. The first recipients undergoing transplantation from 1963 to 1966 were given azathioprine and prednisone. ${ }^{5}$ Since 1966, horse antihuman lymphocyte globulin also has been given during the first several postoperative months ${ }^{3}$ (figure 3 ). Recently cyclophosphamide has been found to be as effective an immunosuppressant in man as azathioprine $^{6}$ and, since it may be less hepatotoxic, has been used in place of azathioprine during the first several postoperative months in the 15 most recent cases in our series (figure 4). For chronic treatment, azathioprine may still be preferable, owing to its less toxic effect on bone marrow.

During the first weeks, prednisone is given in very high doses. Subsequently the 


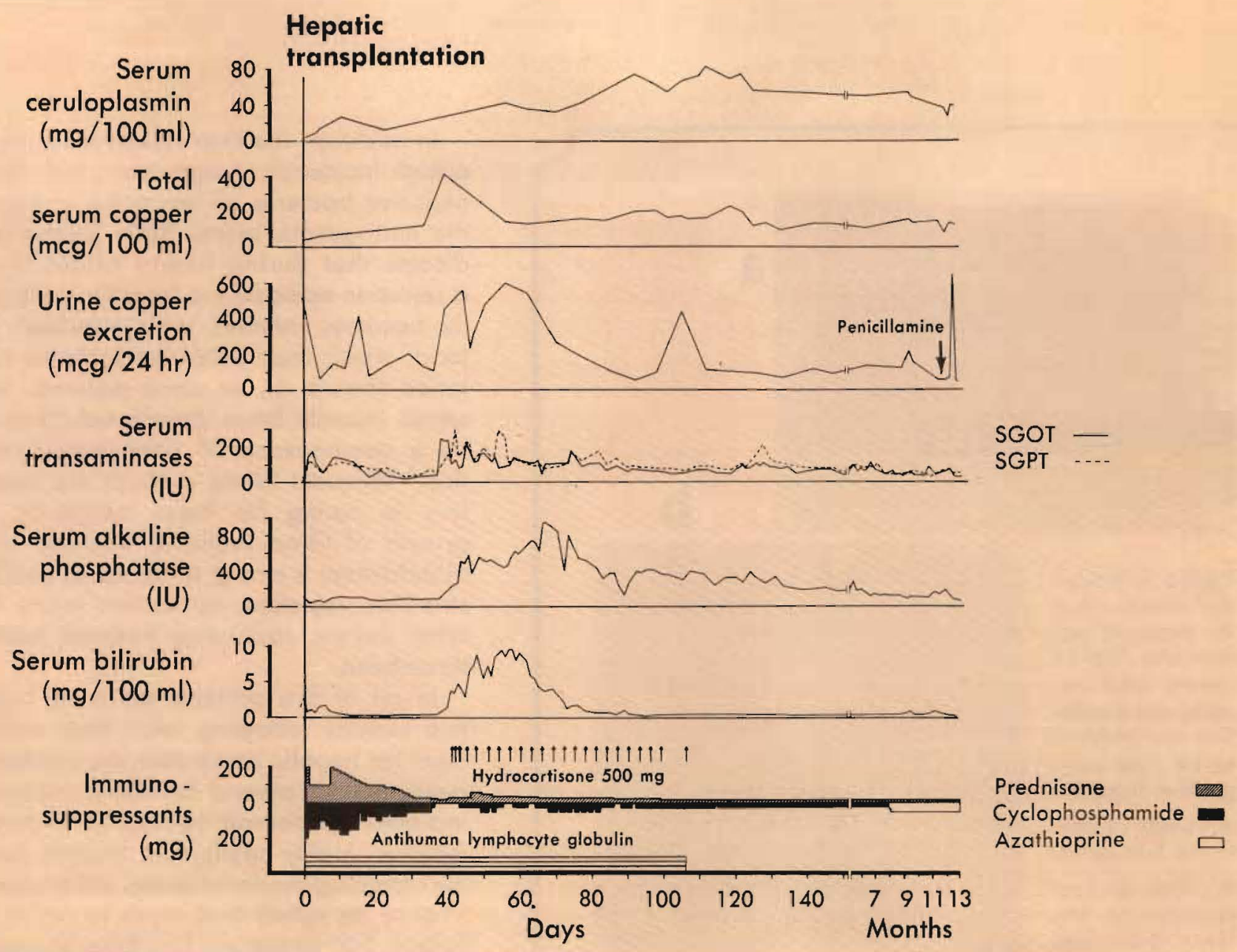

Figure 4. Course of a 14 year old boy with Wilson's disease. Function of the orthotopic hepatic homograft as measured with standard biochemical tests was excellent, except for an early period when bilirubin and alkaline phosphatase rose. At the same time, Australia antigen was present in serum, suggesting serum hepatitis.

Note rapid normalization of serum ceruloplasmin and copper after transplantation. Cyclophosphamide was used as an immunosuppressant initially. After nine months, azathioprine was given instead.

dosage is reduced gradually. If evidence of graft rejection appears, dosage is again increased (figure 3). With time and "graft acceptance," prednisone requirements diminish. The mechanism of this latter phenomenon is poorly understood, but it is a prerequisite of long-term success, as the side effects of prolonged high-dose prednisone use are prohibitive.

Early or late failure to control homograft rejection has been a major cause of death in about one-third of our hepatic homograft recipients. In a few patients a second transplantation was carried out when graft insufficiency due to rejection became severe. One patient survived for 12 months after the second operation.

\section{The Compromised Host}

The dilemma of chronic immunosuppression is to provide enough therapy to prevent rejection, but not so much that the patient dies of immunologic invalidism. In a given case this fine balance may not be achievable. With a frequency equal to that of failure to give enough treatment to prevent rejection, overtreatment has been responsible for infectious complications. 


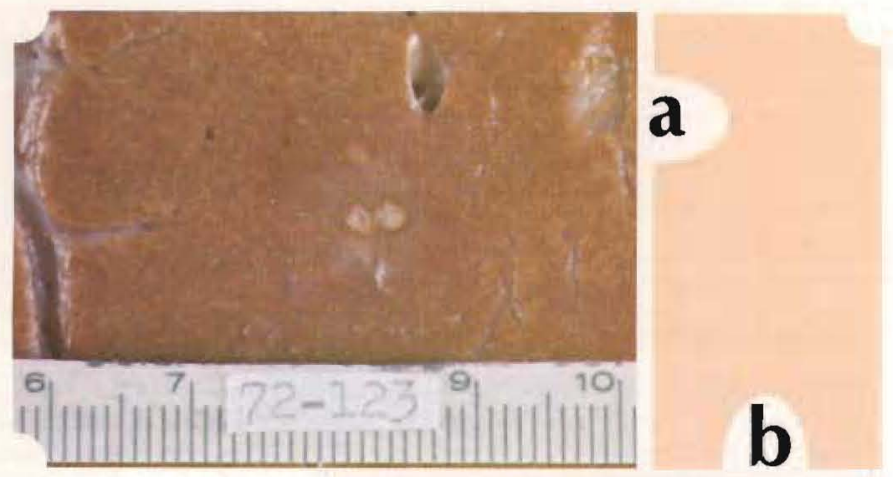

Figure 5. Nocardial infection in a 29 year old patient who died 21 months after hepatic transplantation was performed for chronic aggressive hepatitis.

a. Fungal abscess in the homograft.

b. Nocardia asteroides in the brain. (Brown and Sten stain, $\times 200$.)
In addition, the liver recipients have had a high incidence of septicemia with Gramnegative bacteria of the types existing in the endogenous bowel flora. Evidence indicates that during biliary obstruction or a rejection episode, the hepatic graft readily becomes infected with intestinal bacteria, which then enter the systemic circulation (figure 6). In some patients, large septic infarcts have developed, probably as a consequence of uncontrolled rejection. ${ }^{3}$ Frequent blood cultures are mandatory in caring for these recipients, and growth of Gram-negative bacteria in the bloodstream is strong evidence of graft obstruction, rejection, or serious injury from other causes, including hepatic vascular thrombosis.

In all of five patients surviving beyond two months following total liver replacement for hepatic carcinoma, carcinomatosis developed. In several the tumor recurrence included the hepatic homograft (figure 7). Another highly malignant hepatic tumor, the hemangioendothelioma, infiltrates the liver to an extent that leads to rapid liver failure, but metastasis has been thought to be uncommon. Recently a patient with this disease underwent liver replacement and was found to have widespread metastases within three months.

It is possible that the rapidity and extent

An example of these complications was a recent infection with a fungus, Nocardia, manifested by inflammatory skin nodules in a recipient whose graft had functioned well for 21 months. In spite of three months' treatment with sulfonamides, the patient died with disseminated fungal abscesses involving almost every organ (figure 5). The infecting organism was one of the opportunists that become pathogenic in immunologically compromised hosts. Others are Candida, Aspergillus and Cryptococcus among the fungi; cytomegalovirus, herpes simplex, and varicella-zoster among the viruses; and the nonclassified Pneumocystis carinii. Infections with all these organisms have occurred in our series, and in about a third of the patients the infection has contributed to death. of occurrence of metastases in these patients are a consequence of loss of the "immunologic surveillance" by which mutant cells can be eliminated or restricted in their growth potential. ${ }^{7}$ Be that as it may, the very high incidence of tumor recurrence has dampened, if not eliminated, enthusiasm for liver replacement as treatment of malignant hepatic disease.

\section{Late Results}

Survival beyond one, two and three years has been achieved in 11, 4 and 2 of the patients, respectively (table 1). Nine patients are presently alive, of whom four underwent transplantation more than one year ago, two more than two years ago, and one more than three years ago. 


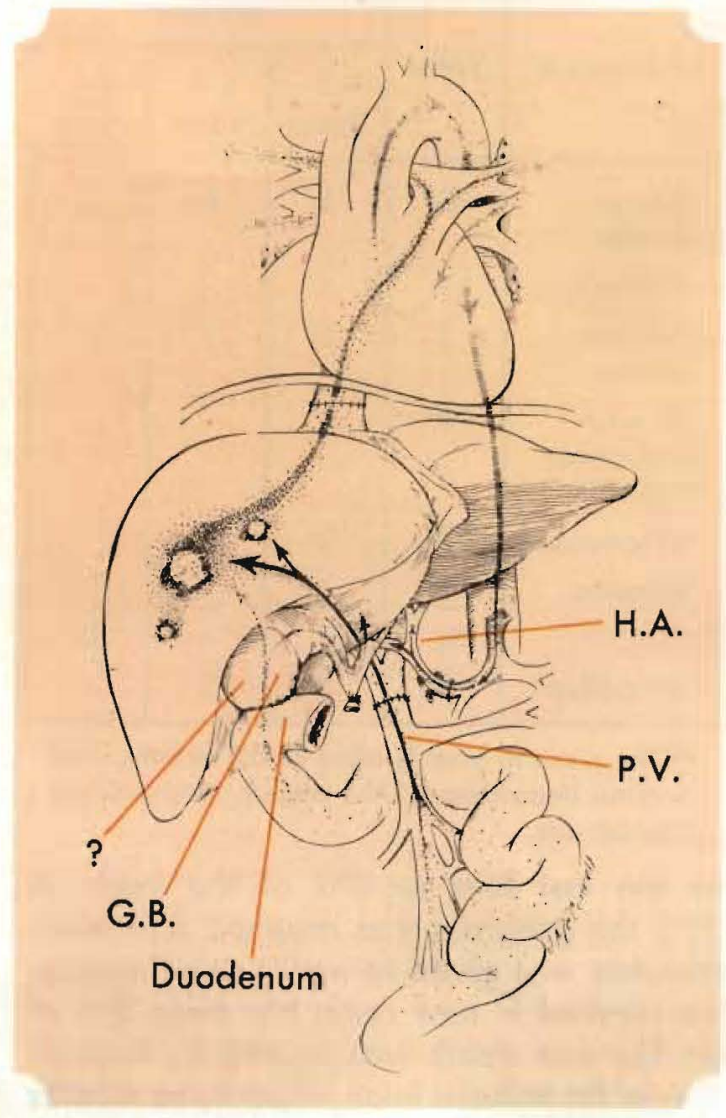

Figure 6. An explanation of the predisposition of liver homograft recipients to bacterial sepsis. Presumably the invading microorganisms enter via the portal vein or the reconstructed biliary tract.

Figures 1 and 6 adapted from Starzl TE: Ann Surg 168:392, 1968.

The largest group of long-term survivors is found among the children treated for biliary atresia. Of 20 patients treated more than 12 months ago, six lived for at least one year. Promising results have been recorded also in the treatment of Wilson's disease. ${ }^{8}$ Two teen-age boys with this disease underwent transplantation 37 and 17 months ago, respectively. One of them was in hepatic failure, and the other had severe neurologic involvement associated with cirrhosis of the liver. Biopsy specimens have shown no accumulation of copper in the grafts, and in the second patient, markedly suppressed serum levels of copper and

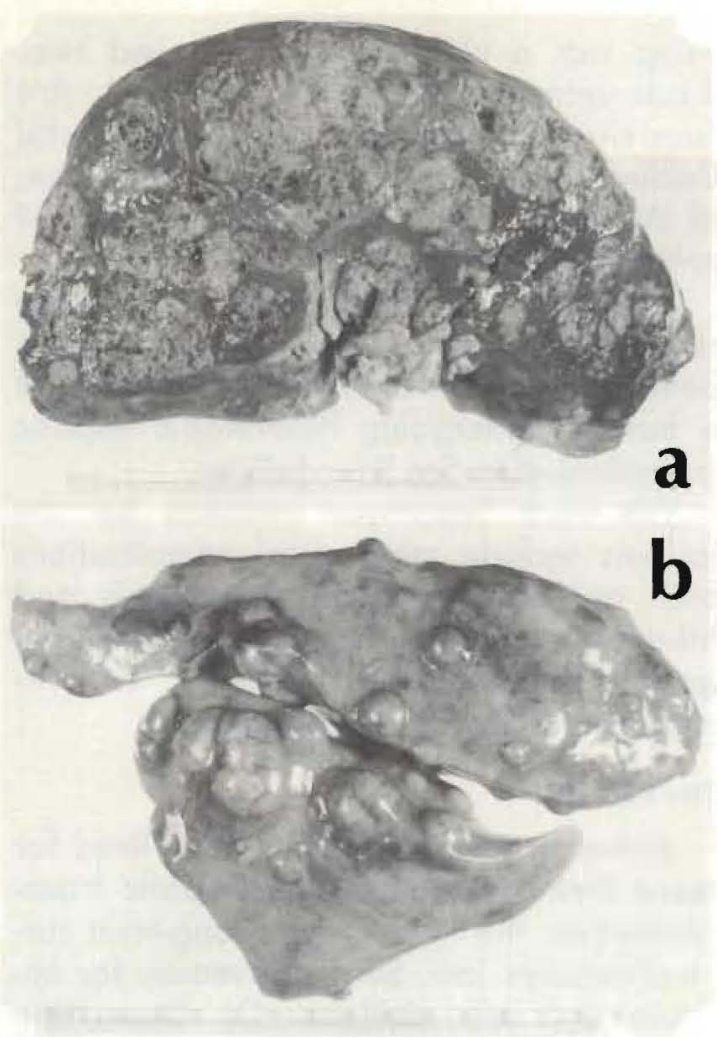

Figure 7. Metastases in a 15 year old patient who died five months after hepatic transplantation for hepatoma.

a. Hepatic homograft has been virtually replaced by tumor.

b. Extensive pulmonary metastases.

Figure 3 adapted from and figure 7 reproduced from Starzl TE, Putnam CW. ${ }^{3}$

ceruloplasmin have normalized (figure 4). The findings are consistent with, but do not prove, the hypothesis that the metabolic defect in Wilson's disease is liver-based. Both patients are alive and well.

Among the patients with benign liver disease, the worst results of transplantation have been in those with Laennec's or biliary cirrhosis, none of whom have survived beyond one year. These results probably reflect the unfavorable case selection mentioned earlier and should not discourage further efforts to operate on such patients.

The only patient with a malignancy in our series in whom metastases did not de- 
velop was a child treated two and twothirds years ago for biliary atresia. In this case a small hepatoma was an incidental finding. Calne, of Cambridge, and Daloze, of Montreal, have each cured one case of primary hepatic malignancy.

Neither in our small series nor among recipients treated at other centers has there been an instance of extended survival of a patient undergoing heterotopic hepatic transplantation for liver failure.

The most important reasons for the failures include mechanical complications such as hepatic arterial thrombosis and biliary obstruction or fistula, uncontrollable graft rejection, severe infections, and recurrence of hepatic malignancy.

\section{The Future}

Although several patients have lived for more than two years after hepatic transplantation, the incidence of long-term survival remains low. Several avenues for improvement are obvious. (1) Operation should be done at an optimum stage, before the patient's condition is agonal. (2) Extensive collaboration within the medical community regarding utilization of organs from patients with brain death would increase the chances of treating more patients at the right time. (3) Careful preoperative evaluation of vascular and biliary anatomy might prevent some of the technical complications. (4) Further improvements in the means for controlling the immune response are crucial.

Even now, a major upgrading of results should be attainable. Because of discouragement with our program in 1971, a moratorium was declared and was in effect
TABLE 1. ORTHOTOPIC HEPATIC TRANSPLANTATION

\begin{tabular}{|c|c|c|c|c|}
\hline \multirow{2}{*}{ Indications } & \multirow{2}{*}{ Total } & \multicolumn{3}{|c|}{ Survivors } \\
\hline & & $\begin{array}{l}1+ \\
\text { Years }\end{array}$ & $\begin{array}{l}2+ \\
\text { Years }\end{array}$ & $\begin{array}{l}3+ \\
\text { Years }\end{array}$ \\
\hline $\begin{array}{l}\text { Biliary } \\
\text { atresia }\end{array}$ & $26^{*}$ & $6+$ & 3 & 1 \\
\hline Cirrhosis & 10 & & & \\
\hline $\begin{array}{l}\text { Wilson's } \\
\text { disease }\end{array}$ & 2 & 2 & 1 & 1 \\
\hline $\begin{array}{l}\text { Chronic } \\
\text { aggressive } \\
\text { hepatitis }\end{array}$ & 5 & 1 & & \\
\hline Carcinoma & 12 & 2 & & \\
\hline \multirow[t]{2}{*}{ Sarcoma } & 1 & - & - & - \\
\hline & 56 & 11 & 4 & 2 \\
\hline Now alive & 9 & 4 & 2 & 1 \\
\hline
\end{tabular}

*One patient, who is alive at $\mathbf{3 2}$ months, had a small hepatoma. TOne patient received two homografts.

for the last four months of the year. In 1972 the program was resumed and extra attention was given to many small details. The survival in new cases has been five of six. The one death was caused by hepatic arterial thrombosis in an infant three months of age whose first homograft, from an anencephalic newborn, was too small and whose second homograft implanted three months later, from a donor five years of age, was too large.

With improved results the indications for hepatic transplantation could be expanded. In addition to the numerous patients suffering from severe destructive hepatic disease, a large number of patients with liver-based errors of metabolism could benefit from the procedure.

Address reprint requests to Dr. Starzl.

\section{REFERENCES}

1. Starzl TE, Porter KA, Andres G, et al: Long-term survival after renal transplantation in humans. Ann Surg 172: 437-472, 1970

2. Dausset J, Rapaport FT: The role of histocompatibility typing in clinical organ transplantation. (Editorial) Transplant Proc 3:979-1122, 1971

3. Starzl TE, Putnam CW: Experience in Hepatic Transplantation. Philadelphia, WB Saunders Company, 1969

4. Martineau G, Porter KA, Corman J, et al: Delayed biliary duct obstruction after orthotopic liver transplantation. Surgery 72:604-610, 1972
5. Starzl TE: Experience in Renal Transplantation. Philadelphia, WB Saunders Company, 1964

6. Starzl TE, Putnam CW, Halgrimson CG, ef al: Cyclophosphamide and whole organ transplantation in human beings. Surg Gynecol Obstet 133:981-991, 1971

7. Starzl TE, Penn I, Putnam $\mathrm{CW}$, et al: latrogenic alterations of immunologic surveillance in man and their influence on malignancy. Transplant Rev 7:112-145, 1971

8. Dubois RS, Rodgerson DO, Martineau G, et al: Orthotopic liver transplantation for Wilson's disease. Lancet 1:505-508, 1971 